(C) 2020 IEEE. Personal use of this material is permitted. Permission from IEEE must be obtained for all other uses, in any current or future media, including reprinting/republishing this material for advertising or promotional purposes, creating new collective works, for resale or redistribution to servers or lists, or reuse of any copyrighted component of this work in other works. 


\title{
Contribution of residual quasiparticles to the characteristics of superconducting thin-film resonators
}

\author{
T. Noguchi, S. Mima, and C. Otani
}

\begin{abstract}
We found that there are a significant number of quasiparticles present in the superconductor even at $T \ll T_{c}$ and that those quasiparticles seriously contribute to the characteristics of superconducting thin-film resonators. The temperature behaviors of the resonator characteristics are well explained by that of the normal conductivity of quasiparticles including the contributions from Kondo effect, phonon, and electron-electron scatterings. The readout microwave power dependence of the resonator characteristics are well explained by the change of quasiparticle density due to the energy re-distribution of quasiparticles under microwave field in the resonator. The resistive loss of the resonator decreases as the power of the readout microwave increases, and when the power of the readout microwave becomes sufficiently large, the resonator loss is dominated by the dielectric loss of the substrate.
\end{abstract}

Index Terms - superconducting resonator, residual resistance, quality factor, Kondo effect, kinetic inductance, Fermi liquid

\section{INTRODUCTION}

W E have been developing Microwave Kinetic Inductance detector (MKID) for future application to large format radio cameras for astronomical observations. Now we are making and studying two kinds of MKIDs; one is based on $\mathrm{Al}$ quarter-wave resonator and the other is $\mathrm{Nb}$ quarter-wave resonator [4], [5]. and their quality factors and resonance frequencies with respect to not only temperature but also readout microwave power are being measured.

When we measured the internal quality factor of a resonator at temperatures well below $T_{c}$, we frequently found that $Q_{i}$ decreases (or $1 / Q_{i}$ increases) as temperature is lowered as shown in Fig. 1 It is interesting that $1 / Q_{i}$ increases as $\log T$, which is shown in the inset of Fig. 1 . Since the $1 / Q_{i}$ is approximately equal to the ratio of residual resistance to total reactance of the resonator, $\log T$ dependence of $1 / Q_{i}$ indicates that residual resistance is proportional to $\log T$, which strongly reminds me of the Kondo effect in the normal metal. The temperature behavior of $1 / Q_{i}$ indicates that there are significant number of quasiparticles present even at $T \ll T_{c}$ and that they behave like electrons in normal metals.

So, at the beginning of this research we made the following assumptions:

Manuscript received September 30, 2018.

T. Noguchi is with the Advanced Technology Center, National Astronomical Observatory, 2-1-1 Osawa, Mitaka, Tokyo, 181-8588 Japan (email: Takashi.Noguchi@nao.ac.jp).

T. Noguchi, S. Mima, and C. Otani are with RIKEN, , 2-1 Hirosawa, Wako, Saitama 351-0198, Japan.

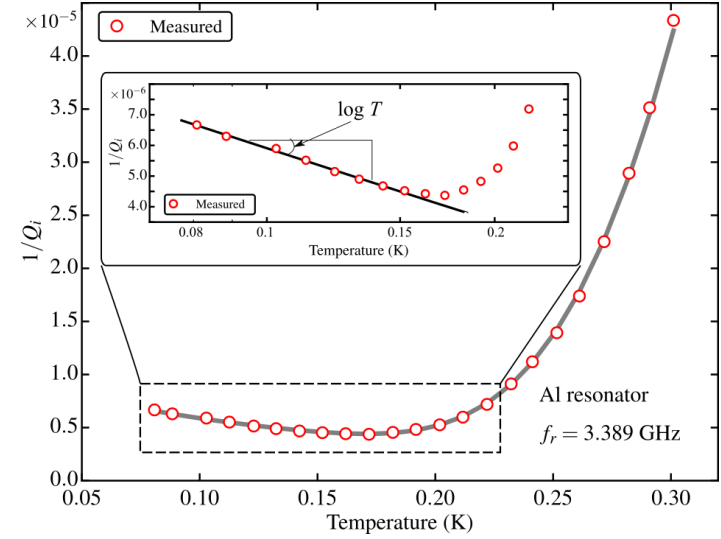

Fig. 1. (a) Measured Internal quality factor $Q_{i}$ (upper panel) and fractional resonance frequency change $\delta f_{r} / f_{r}$ (lower panel) as a function of temperature. Red lines are the calculated ones to fit the measured data. (b) $\log T$ dependence of fractional resonance frequency change $\delta f_{r} / f_{r}$.

1) There are significant number of quasiparticles in the superconductor even at temperatures well below $T_{c}$.

2) Those residual quasiparticles in the superconductor behave like electrons in a normal metal under a microwave field.

3) The residual resistance of quasiparticles in the superconductor depends not only on temperature but also on readout microwave power even at the frequency much smaller than the gap frequency.

Then, we measured and analyzed the temperature dependence of the internal quality factor and the resonance frequency using a fitting method based on both the extended MattisBardeen theory [1], [6] and the residual resistance taking into account the contribution of scatterings of the quasiparticles at low temperature. In this paper, it will be shown that the temperature behavior of not only the internal quality factor but also the resonance frequency observed in the $\mathrm{Al}$ and $\mathrm{Nb}$ film resonators are determined by the residual resistance contributed from quasiparticle scatterings such as the Kondo effect at low temperature.

It will be also shown that the energy redistribution of the residual quasiparticles is induced due to a strong microwave field in the resonator, so that the number of quasiparticles near the Fermi level, which mainly contribute to the normal conductivity, decreases with increasing the microwave field. It will be shown that the increase of the internal quality factor of 


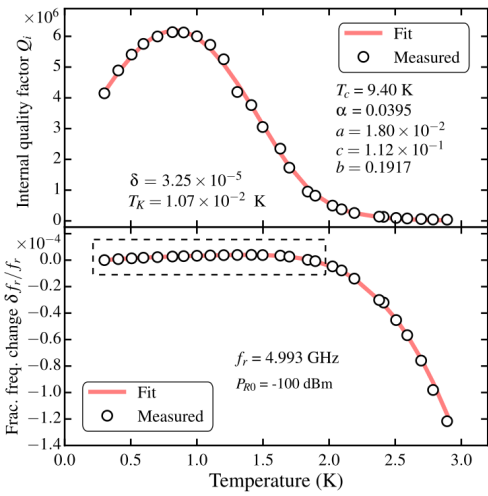

(a)

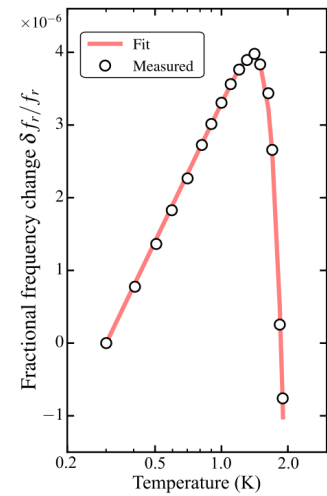

(b)
Fig. 2. (a) Measured Internal quality factor $Q_{i}$ (upper panel) and fractional resonance frequency change $\delta f_{r} / f_{r}$ (lower panel) as a function of temperature. Red lines are the calculated ones to fit the measured data. (b) $\log T$ dependence of fractional resonance frequency change $\delta f_{r} / f_{r}$.

the resonator with increasing readout microwave power might be attributed to the energy redistribution of the quasiparticles under the strong microwave field in the resonator. Finally, it will be mentioned that the loss of the resonator at the high readout microwave power is dominated by the contribution of the dielectric loss of the substrate.

\section{EXPERIMENTAL}

Quarter wavelength coplanar waveguide (CPW) type thinfilm resonators using $\mathrm{Al}$ and $\mathrm{Nb}$ film were made onto a $4 \times 20$ $\mathrm{mm}^{2} \mathrm{Si}$ and sapphire chips respectively. The width of a center line of the CPW and the gap between the center line and the ground conductor were 8 and $3 \mu \mathrm{m}$, respectively. A microwave signal in the 3.8 or $4.9 \mathrm{GHz}$ band is transmitted from one port of the CPW feed line (readout line) to the other port and the transmission coefficient $S_{21}$ was measured as a function of temperature and frequency using a vector network analyzer (VNA).

It has been shown [2], [9] that both the decrease of the $Q_{i}$ and the broad peak of the $\delta f_{r} / f_{r}$ at low temperature in the $\mathrm{Nb}$ resonator can be well explained by the increase of residual resistance of quasiparticles due to the Kondo effect [3]. Light red lines in upper and lower panel of the left figure in Fig. 2 are fitting curves calculated by the eqs. (4) and (5) in Ref. [9] taking into account the contributions of the Kondo effect, phonon scattering and electron-electron scattering to the residual resistance of the quasiparticles. It should be also noted here that the broad peak of $\delta f_{r} / f_{r}$ around $1.5 \mathrm{~K}$ is caused by the contribution of the kinetic inductance of the residual quasiparticles.

From the fact that both the measured $1 / Q_{i}$ and $\delta f_{r} / f_{r}$ agree very well with those calculated by taking into account the contribution of the residual resistance of quasiparticles at low temperature, we confirmed that a significant number of quasiparticles still remain in the superconducting gap at low temperature and that they behaves like the electrons in normal metals.

Not only the temperature but also the readout microwave power dependences of $Q_{i}$ and $\delta f_{r} / f_{r}$ of a $\mathrm{Nb}$ resonator are

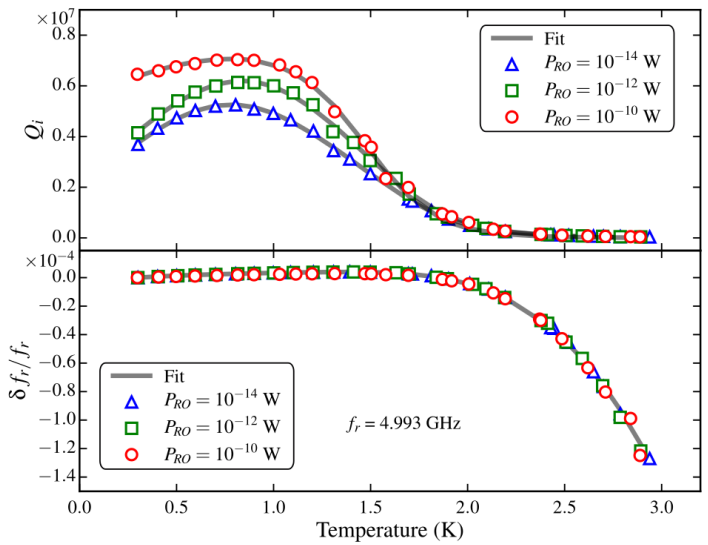

Fig. 3. Internal quality factor $Q_{i}$ (upper panel) and resonance frequency $f_{r}$ (lower panel) of the $\mathrm{Nb}$ resonator as a function of temperature for different readout microwave power $P_{R O}$.

shown in Fig. 3 It is shown that our approximate expressions for $Q_{i}$ and $\delta f_{r} / f_{r}$ are well fit to the measured ones for different readout microwave power. Interesting point of this plot is that $Q_{i}$ strongly depends on readout microwave power, whereas $\delta f_{r} / f_{r}$ is almost independent of the readout microwave power. Very similar behavior of $Q_{i}$ and $f_{r}$ with respect to the readout microwave power $P_{R O}$ was also observed in the $\mathrm{Al}$ resonator as shown in the upper and lower panel in Fig. 4, respectively. It should be noted here that the $Q_{i}$ of the $\mathrm{Al}$ resonator changes more than factor of 10 in magnitude in the similar power range of $P_{R O}$ at the lowest measured temperature., whereas $f_{r}$ does not depend on the readout microwave power $P_{R O}$. In the next section, we will discuss the reason why $Q_{i}$ increases with increasing readout microwave power while the resonance frequency remains constant.

\section{READOUT MICROWAVE POWER DEPENDENCE}

It is clearly shown by Fig. 4 that the resonance frequency $f_{r}$ for the $\mathrm{Nb}$ and $\mathrm{Al}$ resonator are almost constant and independent of the readout microwave power $P_{R O}$. Assuming that the microwave properties of a superconductor can be described by a complex conductivity, $\sigma=\sigma_{1}-i \sigma_{2}$, this means that the $\sigma_{2}$ of the superconducting $\mathrm{Al}$ and $\mathrm{Nb}$ are approximately unchanged against the $P_{R O}$ because the resonance frequency $f_{r}$ is determined only by $\sigma_{2}$. On the other hand, the internal quality factor $Q_{i}$ of the $\mathrm{Al}$ resonator are strongly dependent on the readout microwave power $P_{R O}$. It is found that the internal quality factor $Q_{i}$ of the $\mathrm{Nb}$ resonator also increases as the readout microwave power $P_{R O}$ increases. This means that the $\sigma_{1}$ decreases as the readout microwave power $P_{R O}$ increases in this power range. Because the internal quality factor $Q_{i}$ of a resonator is described by a ratio of the $\sigma_{2}$ and $\sigma_{1}$ of the superconductor and the $\sigma_{2}$ is constant against the readout microwave power $P_{R O}$ in this power range as mentioned above.

According to the Drude model [11], the quasiparticle current $\mathbf{J}_{q p}$ in the microwave electric field $\mathbf{E}$ is a function of the conductivity $\sigma_{1}$ as

$$
\mathbf{J}_{q p}=\frac{n_{q p} e^{2} \tau}{m} \mathbf{E}=\sigma_{1} \mathbf{E}
$$




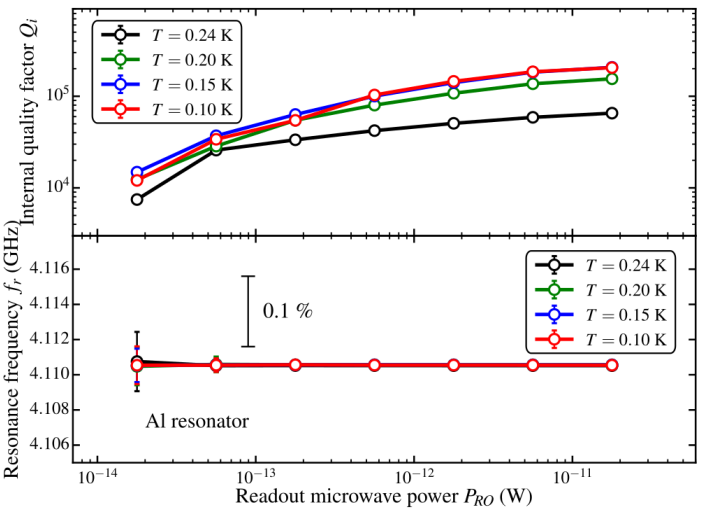

Fig. 4. Internal quality factor $Q_{i}$ (upper panel) and resonance frequency $f_{r}$ (lower panel) of the $\mathrm{Al}$ resonator as a function of readout microwave power $P_{R O}$ for different temperatures.

where $\mathbf{E}$ is an amplitude of the microwave field, $n_{q p}$ is the number of quasiparticles, $\tau$ is a scattering time, and $m$ and $e$ are the electron mass and charge, respectively. The conductivity $\sigma_{1}$ is proportional to the number of quasiparticle $n_{q p}$ as

$$
\sigma_{1}=\frac{n_{q p} e^{2} \tau}{m}
$$

According to eq. 22, the the inverse quality factor $1 / Q_{i}$ of a superconducting resonator is thought to be proportional to the quasiparticle density $n_{q p}$, since the $1 / Q_{i}$ is proportional to the conductivity $\sigma_{1}$ as described above. Therefore, we calculated the number of quasiparticles under the microwave field in the resonator, assuming that the number of quasiparticles $n_{q p}$ in the resonator changes according to the readout microwave power $P_{R O}$.

Following Tien and Gordon's argument [12], we start with a very simple case that a microwave electric field is excited between the center conductor and the ground plane of the CPW. In this case, the microwave electric field sets up a potential difference $V_{\text {res }} \cos \omega t$ between them.

When no field is present the wave functions of the charge carriers of energy $\mathscr{E}$ satisfy the unperturbed Hamiltonian $H_{0}$

$$
\psi(\mathbf{r}, t)=\psi_{0}(\mathbf{r}) \exp \left(-i \frac{\mathscr{E} t}{\hbar}\right)
$$

The perturbed Hamiltonian due to the oscillating electric field is given by

$$
H=H_{0}+e V_{\text {res }} \cos \omega t
$$

This interaction Hamiltonian only effects the time-dependent part of the wave function.

The new wave function under influence of the microwave field becomes

$$
\begin{aligned}
\psi(\mathbf{r}) & =\psi_{0}(\mathbf{r}) \exp \left\{-i \frac{\mathscr{E} t}{\hbar}-\frac{i}{\hbar} \int_{0}^{t} e V_{\text {res }} \cos \left(\omega t^{\prime}\right) d t^{\prime}\right\} \\
& =\psi_{0}(\mathbf{r}) \exp \left(-i \frac{\mathscr{E} t}{\hbar}\right) \sum_{-\infty}^{\infty} J_{n}(\xi) \exp (-i n \omega t)
\end{aligned}
$$

where $J_{n}(\xi)$ is the $n$-th order Bessel function of the first kind and $\xi=\frac{e V_{\text {res }}}{\hbar \omega}$ is an amplitude of the normalized microwave

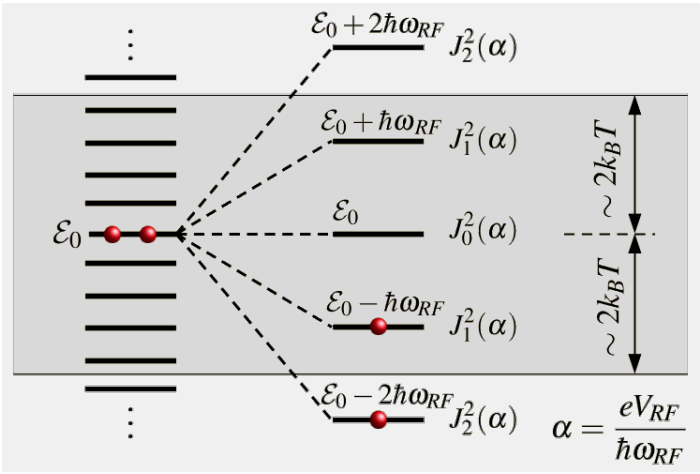

Fig. 5. Development of sidebands of the original energy as a consequence of the microwave field. The hatched region corresponds to the tail of the Fermi distribution due to the thermal broadening.

voltage. The wavefunction of a quasiparticle is modulated by the perturbation of $e V r f \cos \omega t$ and a quasiparticle state split into many levels with a energy spacing $\hbar \omega$ and an amplitude Bessel function $J_{n}(\xi)$. Accordingly, the effective density of states in the presence of the microwave field becomes

$$
D^{\prime}(\mathscr{E})=\sum_{n=-\infty}^{\infty} D_{s}(\mathscr{E}+n \hbar \omega) J_{n}^{2}(\xi),
$$

where $D_{s}(\mathscr{E})$ is an unperturbed density of states in a superconductor given by

$$
D_{s}(\mathscr{E})=\left|\frac{\mathscr{E}}{\sqrt{\mathscr{E}^{2}-\Delta^{2}}}\right| .
$$

where $\Delta$ is a complex gap energy of the superconductor, i.e. $\Delta=\Delta_{1}+i \Delta_{2}$ and $\frac{\Delta_{2}}{\Delta_{1}} \ll 1$ and $\Delta_{1}$, and $\Delta_{2}$ are real numbers, respectively [1].

When split multi-levels are generated, some quasiparticles transition to lower energy levels, resulting in a change in the energy distribution of the quasiparticles and in a decrease of the number of quasiparticles occupying the tail of the Fermi function. The quasiparticle density under microwave field is given by

$$
\begin{aligned}
n_{q p}(x)= & 4 N_{0} \int_{0}^{\infty} D_{s}(\mathscr{E}) f(\mathscr{E}) d \mathscr{E} \\
= & n_{q p}(0) J_{0}^{2}(x)+4 N_{0} \sum_{n=1}^{\infty} J_{n}^{2}(\xi) \\
& \times \int_{0}^{\infty}\left[D_{s}(\mathscr{E}-n \hbar \omega)+D_{s}(\mathscr{E}+n \hbar \omega)\right] f(\mathscr{E}) d \mathscr{E}
\end{aligned}
$$

where $n_{q p}(0)$ is a quasiparticle density in the thermal equilibrium is defined by

$$
n_{q p}(0)=4 N_{0} \int_{0}^{\infty} D_{s 0}(\mathscr{E}) f(\mathscr{E}) d \mathscr{E},
$$

where $f(\mathscr{E})$ is the Fermi function. When the photon energy of the readout microwave is much smaller than the gap energy $\left(\frac{\hbar \omega}{\Delta} \ll 1\right)$, which is satisfied in most MKID resonators, the following approximation can be applied for small $n$

$$
D_{s}(\mathscr{E} \pm n \hbar \omega) \approx D_{s}(\mathscr{E}) \pm(n \hbar \omega) \frac{d D_{s}(\mathscr{E})}{d \mathscr{E}}
$$




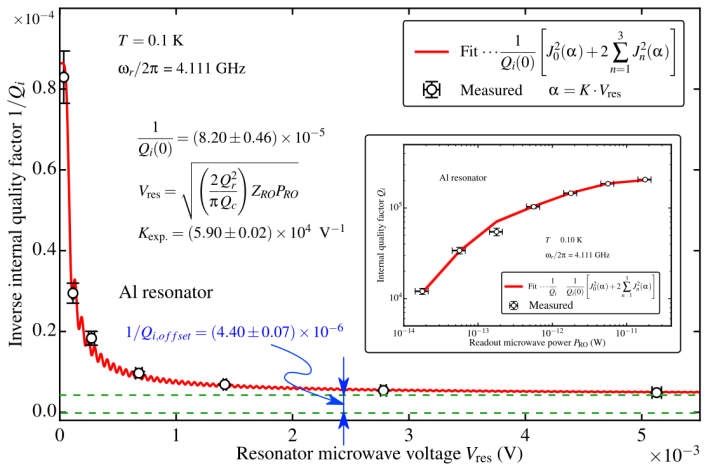

Fig. 6. The inverse internal quality factors of $1 / Q_{i}$ of the $\mathrm{Al}$ resonator at $T=0.1 \mathrm{~K}$ are shown as a function of microwave voltage in the transmissionline resonator, $V_{\text {res }}$. The solid red line is calculate by eq. [11. Inset is a plot of $Q_{i}$ as a function of the readout microwave power, $P_{R O}$.

Energy redistribution of quasiparticles is caused by transitions between quasiparticle levels involving the absorption and emission of multiple photons. Since the probability of such multiple-photon transitions decreases rapidly as the number of photons increases, the terms with $n \leq 4$ in eq. (8) may be omitted. The

$$
n_{q p}(\xi) \simeq n_{q p}(0)\left[J_{0}^{2}(\xi)+2 \sum_{n=1}^{n=3} J_{n}^{2}(\xi)\right] .
$$

Considering that the inverse quality factor $1 / Q_{i}$ is proportional to the quasiparticle density $n_{q p}(\xi)$, eq. 111) shows that the inverse quality factor $1 / Q_{i}$ varies as a function of the sum of $J_{n}^{2}(\xi)$ with respect to the normalized microwave voltage $\xi$. This indicates that quasiparticle energy redistribution occurs in the presence of the microwave field, resulting in a decrease in the number of quasiparticles with energy within $\pm k_{B} T$ from the Fermi level.

Since the internal microwave power, $P_{I N T}$, in a resonator is related to the readout microwave power $P_{R O}$ by [13]

$$
P_{I N T}=\left(\frac{2}{\pi} \frac{Q_{r}^{2}}{Q_{c}}\right) P_{R O},
$$

an amplitude of the microwave voltage $V_{\text {res }}$ in the resonator transmission line is calculated as

$$
V_{\text {res }}=\sqrt{\left(\frac{2}{\pi} \frac{Q_{r}^{2}}{Q_{c}}\right) Z_{R O} P_{R O}}
$$

where $Q_{c}$ and $Q_{r}$ are the coupling and loaded quality factor of the resonator, respectively, and $Z_{R O}$ and $Z_{\text {res }}$ are characteristic impedance of a readout transmission line and the resonator transmission line, respectively and are assumed to be $Z_{\text {res }}=$ $Z_{R O} \simeq 50 \Omega$.

In Fig. 6, measured inverse internal quality factor $1 / Q_{i}$ of an $\mathrm{Al}$ resonator at $0.1 \mathrm{~K}$ is shown as a function of the microwave voltage in the resonator calculate from the measured readout microwave power $P_{R O}$ using eq. 13 .

Note here that measured $1 / Q_{i}$ seems, however, to have an offset of $1 / Q_{i, \text { offset }} \approx 4.40 \times 10^{-6}$ which is approximately only $5 \%$ of $1 / Q_{i}$ for the lowest readout microwave power, and seems to be insensitive and constant against the microwave
TABLE I

MEASURED $1 / Q_{i, o f f s e t}$ AND $\tan \delta$ FOR SI AND SAPPHIRE SUBSTRATE

\begin{tabular}{l|l|c}
\hline Substrate material & $1 / Q_{i, o f f s e t}$ (Measured) & $\tan \delta$ (Reported) \\
\hline \hline High resistivity Si & $\begin{array}{l}1.09 \times 10^{-6} \\
4.40 \times 10^{-6}\end{array}$ & $0.3-5 \times 10^{-6}|14|$ \\
\hline Crystalline sapphire & $\begin{array}{l}2.43 \times 10^{-8} \\
1.38 \times 10^{-7}\end{array}$ & $0.8-5 \times 10^{-7}[15$ \\
\hline
\end{tabular}

voltage in the resonator. Then we subtracted the constant offset from the measured $1 / Q_{i}$ and fit to the subtracted data was made using eq. 11. The normalized voltage $\xi$ in the resonator is experimentally determined from the relation, $\xi=K \cdot V_{\text {res }}$, where $K$ is a scaling factor which is theoretically given by $\frac{e}{\hbar \omega_{r}}=5.87 \times 10^{4} \mathrm{~V}^{-1}$, where $\omega_{r}$ is a resonance frequency of the resonator. It is clearly demonstrated in Fig 6 that the measured data agree well to the calculated one using eq. (11). From the fit we obtained the scaling parameter of $K=(5.90 \pm 0.02) \times 10^{4} V^{-1}$ which is only a factor 2 different from the theoretical value, which is quite consistent with the theoretical value of $\frac{e}{\hbar \omega_{r}}=5.87 \times 10^{4} \mathrm{~V}^{-1}$. A very similar result of inverse quality factor $1 / Q_{i}$ as a function of the normalized microwave voltage in the resonator is obtained in the $\mathrm{Nb}$ resonator measured at $0.8 \mathrm{~K}$. These fit results indicate that the quasiparticle energy redistribution is caused by a strong microwave field in the resonator, so that the number of quasiparticles near the Fermi level, which mainly contribute to the normal conductivity $\sigma_{1}$, decreases with increasing the amplitude of the microwave field.

\section{Dielectric loss OF THE SUbSTRATE}

Since the resonator used in the experiments is made of a quarter-wavelength $\mathrm{CPW}$ transmission line, the internal quality factor of the resonator, $Q_{i}$ is approximately equal the quality factor of the transmission line, $Q_{T L}$. From the viewpoint of the superconducting transmission line, the inverse of the quality factor of the transmission line, $1 / Q_{T L}$ is given by the sum of the inverse of conductor and dielectric quality factors as

$$
\frac{1}{Q_{T L}}=\frac{R_{s}}{\omega_{r} L}+\frac{G_{d}}{\omega_{r} C_{d}} \approx \frac{1}{Q_{S C}}+\tan \delta
$$

where $R_{s}$ and $\omega_{r} L$ are the surface resistance and reactance of the superconductor, respectively, $Q_{S C}$ is a quality factor of the superconductor, and $G_{d}$ and $\omega_{r} C_{d}$ and $\tan \delta$ are the conductance, susceptance and loss tangent of the dielectric, respectively. When the readout microwave power $P_{R O}$ is sufficiently high, the inverse quality factor of the superconductor, $1 / Q_{S C}$ approaches 0 and inverse transmission-line $Q_{T L}$ is dominated by the inverse dielectric quality factor, $\frac{\widetilde{G}_{d}}{\omega_{r} C_{d}}$ or $\tan \delta$, which should be in close agreement with the $1 / Q_{i, \text { offset }}$ obtained in the experiment,

$$
\frac{1}{Q_{i, o f f s e t}} \approx \frac{G_{d}}{\omega_{r} C_{d}} \approx \tan \delta
$$

The values of $1 / Q_{i, \text { offset }}$ obtained for two $\mathrm{Nb}$ resonators on a sapphire substrate and and two $\mathrm{Al}$ resonators on a $\mathrm{Si}$ substrate, respectively, are shown in Table I In Table I $\tan \delta$ 
of the sapphire and $\mathrm{Si}$ substrate measured at extremely low temperatures and reported are also shown. It is found that the measured $1 / Q_{i, o f f s e t}$ for $\mathrm{Nb}$ and $\mathrm{Al}$ resonators obtained in our experiments are very consistent with reported loss tangent $(\tan \delta)$ for $\mathrm{Si}$ and sapphire substrates measured at extremely low temperatures.

\section{SUMMARY}

It is demonstrated that there are a significant number of quasiparticles present in the superconductor even at $T \ll T_{c}$ and that those quasiparticles seriously contribute to the characteristics of superconducting thin-film resonators.

It is found that temperature behaviors of the resonator characteristics are well explained by that of the normal conductivity of quasiparticles including the contributions from Kondo effect, phonon, and electron-electron scatterings. It is found that readout microwave power dependence of the resonator characteristics are well explained by the change of quasiparticle density due to the energy re-distribution of quasiparticles under microwave field in the resonator.

The conductive loss of the resonator decreases as the power of the readout microwave increases, and when the power of the readout microwave becomes sufficiently large, the resonator loss is dominated by the dielectric loss of the substrate.

\section{REFERENCES}

[1] T. Noguchi, M. Naruse, M. Sekine, K. Karatsu and Y. Sekimoto, IEEE Trans. Appl. Supercond., 26, 1500804, 2016.

[2] T. Noguchi, A. Dominjon and Y. Sekimoto, IEEE Trans. Appl. Supercond., 28, 2400206, 2018.

[3] J. Kondo, Progr. Theor. Phys., 32, 37, 1964

[4] P. Day, H. Leduc, B. Mazin, A. Vayonakis and J. Zmuidzinas, Nature, 425, 817, 2003

[5] B. A. Mazin, B. Bumble, P. K. Day, M. E. Eckart, S. Golwala, J. Zmuidzinas and F. A. Harrison, Appl. Phys. Lett., 89, 222507, 2006.

[6] D. C. Mattis and J. Bardeen, Phys. Rev., 111, 412, 1958.

[7] R. Barends, J. J. A. Baselmans, J. N. Hovenier, J. R. Gao, S. J. C. Yates, T. M. Klapwijk and H. F. C. Hoevers, IEEE Trans. Appl. Supercond., 17, 263, 2007

[8] M. Roesch et al., Proc. 21st Int. Symp. Space Teraherz Tech., 72, 2010.

[9] T. Noguchi, A. Dominjon, M. Kroug, S. Mima and C. Otani, IEEE Trans. Appl. Supercond., 29, 2400205, 2019.

[10] A. Dominjon et al., Proc. 16th International Superconductive Electronics Conference (ISEC), DOI: 10.1109/ISEC.2017.8314192, 2017.

[11] N. Ashcroft and D. Mermin, Solid State Physics (1st ed.), Fort Worth Saunders College, Chapter 1, pp 1-28, 1976.

[12] P. K. Tien and J. P. Gordon, Phys. Rev., 129, 647, 1963.

[13] N. C. Czakon et al., Proc. SPIE - The International Society for Optical Engineering, 7741 , DOI: 10.1117/12.857866, 2010.

[14] A. D. O'Connell et al., Appl. Phys. Lett., 92, 112903, 2008.

[15] J. Krupka, K. Derzakowski, M. Tobar, J. Hartnett, and R. G. Geyer, Meas. Sci. Technol., 10, pp. 387-392. 1999. 